\title{
1-2-3-flavor color superconductivity in compact stars
}

\author{
David Blaschke ${ }^{1,2}$, Fredrik Sandin ${ }^{3}$ and Thomas Klähn ${ }^{4}$ \\ ${ }^{1}$ Institute for Theoretical Physics, University of Wroclaw, 50-204 Wroclaw, Poland \\ 2 Bogoliubov Lab. for Theoretical Physics, JINR Dubna, 141980 Dubna, Russia \\ 3 IFPA, Département AGO, Université de Liège, 4000 Liège, Belgium \\ ${ }^{4}$ Physics Division, Argonne National Laboratory, Argonne, IL 60439, USA \\ E-mail: blaschke@ift.uni.wroc.pl
}

\begin{abstract}
We suggest a scenario where the three light quark flavors are sequentially deconfined under increasing pressure in cold asymmetric nuclear matter as, e.g., in neutron stars. The basis for our analysis is a chiral quark matter model of NambuJona-Lasinio (NJL) type with diquark pairing in the single flavor color-spin-locking (CSL), two-flavor (2SC) and three-flavor color-flavor locking (CFL) channels, and a Dirac-Brueckner Hartree-Fock (DBHF) approach in the nuclear matter sector. We find that nucleon dissociation sets in at about the saturation density, $n_{0}$, when the downquark Fermi sea is populated (d-quark dripline) due to the flavor asymmetry imposed by $\beta$-equilibrium and charge neutrality. At about $3 n_{0}$ u-quarks appear forming a twoflavor color superconducting (2SC) phase, while the s-quark Fermi sea is populated only at still higher baryon density. The hybrid star sequence has a maximum mass of $2.1 \mathrm{M}_{\odot}$. Two- and three-flavor quark matter phases are found only in gravitationally unstable hybrid star solutions.
\end{abstract}

Introduction - Recent results from observations of compact star properties provide constraints on the nuclear equation of state (EoS) [1]. In particular, the high masses $M \sim 2.0 M_{\odot}$ of compact stars in low-mass X-ray binaries, e.g., 4U 1636-536 [2] and the indicated large radius $R>12 \mathrm{~km}$ of the isolated neutron star RX J1856.5-3754 [3] point to a stiff EoS at high densities. Other limits on the stiffness of the EoS comes from heavy-ion collision data for kaon production and elliptic flow (see [1] for references). A key question is whether the phase transition to quark matter can occur inside compact stars [4] and if it is accompanied by observable signatures. Based on a DBHF approach in the nuclear matter sector and a chiral quark matter model with a vector meanfield interaction a recently developed class of hybrid EoS [5, 6] obtained stable hybrid stars with masses from $1.2 \mathrm{M}_{\odot}$ up to $2.1 \mathrm{M}_{\odot}$ in accordance with modern mass-radius constraints. Under the $\beta$-equilibrium condition in compact stars the chemical potentials of quarks and electrons are related by $\mu_{d}=\mu_{s}$ and $\mu_{d}=\mu_{u}+\mu_{e}$. The mass difference between the strange and the light quark flavors $m_{s}>m_{u}, m_{d}$ has two important consequences: (1) the down and strange quark densities are different so charge neutrality requires a finite electron density and, consequently, (2) $\mu_{d}>\mu_{u}$.

When increasing the baryochemical potential, the d-quark chemical potential is the first to reach a critical value where the the partial density of free d-quarks becomes 
finite in a first order phase transition. Due to the finite value of $\mu_{e}$ the u-quark chemical potential is below the critical value and the s-quark density is zero due to the high squark mass. A single-flavor d-quark phase therefore forms. We discuss this phase here under the natural assumption that the neutralizing background is provided by nuclear matter, for details see [7.
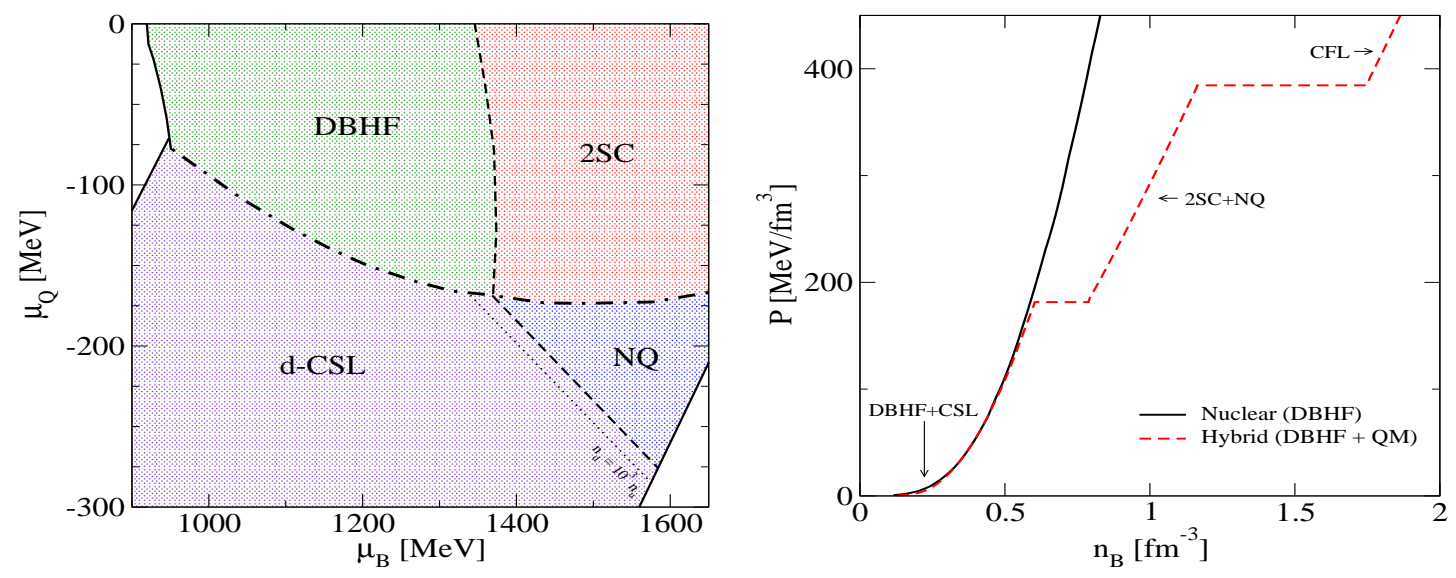

Figure 1. Left panel: Phase diagram in the plane of baryon and charge chemical potential. The dash-dotted line denote the border between oppositely charged phases. Right panel: Pressure of matter in $\beta$-equilibrium as a function of the density.

Phase transition to quark matter: nucleon dissociation - The task to describe the phase transition from nuclear matter to quark matter as a dissociation of three-quark bound states into their quark constituents (Mott transition) has not yet been solved. Only some aspects have been considered yet, e.g., within a nonrelativistic potential model [8] or within the NJL model [9]. We will consider a chemical equilibrium of the type $n+n \leftrightarrow p+3 d$, which results in a mixed phase of nucleons and down quarks once the d-quark chemical potential exceeds the critical value. This scenario is analogous to the dissociation of nuclear clusters in the crust of neutron stars (neutron dripline) and the effect may therefore be called the d-quark dripline. We approximate the quark and nucleon components as subphases described by separate models, e.g., by the DBHF approach and a three-flavor quark model of NJL type [10, 11, 12, 13. The partition function for the latter is given in path-integral representation by

$$
\begin{aligned}
& Z(T, \hat{\mu})=\int \mathcal{D} \bar{q} \mathcal{D} q \exp \left\{\int_{0}^{\beta} d \tau \int d^{3} x\left[\bar{q}\left(i \not \partial-\hat{m}_{0}+\hat{\mu} \gamma^{0}\right) q+\mathcal{L}_{\text {int }}\right]\right\}, \\
& \mathcal{L}_{\text {int }}=G_{S}\left\{\sum_{a=0}^{8}\left[\left(\bar{q} \tau_{a} q\right)^{2}+\left(\bar{q} i \gamma_{5} \tau_{a} q\right)^{2}\right]+\eta_{D 0} \sum_{A=2,5,7} j_{D 0, A}^{\dagger} j_{D 0, A}+\eta_{D 1} j_{D 1}^{\dagger} j_{D 1}\right\},
\end{aligned}
$$

where $\hat{\mu}=\frac{1}{3} \mu_{B}+\operatorname{diag}_{f}\left(\frac{2}{3},-\frac{1}{3},-\frac{1}{3}\right) \mu_{Q}+\lambda_{3} \mu_{3}+\lambda_{8} \mu_{8}$ is the diagonal quark chemical potential matrix and $\hat{m}_{0}=\operatorname{diag}_{f}\left(m_{u}^{0}, m_{d}^{0}, m_{s}^{0}\right)$ the current-quark mass matrix. For $a=0, \tau_{0}=\sqrt{2 / 3} \mathbf{1}_{f}$, otherwise $\tau_{a}$ and $\lambda_{a}$ are Gell-Mann matrices acting in flavor and color spaces, respectively. $C=i \gamma^{2} \gamma^{0}$ is the charge conjugation operator and 
$\bar{q}=q^{\dagger} \gamma^{0}$. The coupling strength, $G_{S}$, of the scalar quark-antiquark current-current interaction and the 3-momentum cutoff, $\Lambda$, are fixed by low-energy QCD phenomenology (see table I of [14]). The relative coupling strengths of the spin-0 and spin-1 diquark currents, $j_{D 0, A}=q^{T} i C \gamma_{5} \tau_{A} \lambda_{A} q$ and $j_{D 1}=q^{T} i C\left(\gamma_{1} \lambda_{7}+\gamma_{2} \lambda_{5}+\gamma_{3} \lambda_{2}\right) q$, are essentially free parameters, but we restrict the discussion to the values from a Fierz transformation of the one-gluon exchange interaction, $\eta_{D 0}=3 / 4$ and $\eta_{D 1}=3 / 8$, see [15]. The mass gaps, pairing gaps and quark matter EoS are obtained from the mean-field thermodynamic potential, $\Omega_{M F}=-T \ln Z_{M F}$, which is obtained from (11). In Fig. 1 the phase diagram and hybrid EoS are illustrated. The phase diagram maps the phases with lowest free energy in the plane of baryon chemical potential, $\mu_{B}$, and electric charge chemical potential, $\mu_{Q}$. The EoS is the path between oppositely charged phases. Volume fractions are determined such that the mixture of the two phases is neutral. The equations for the mass gaps and pairing gaps are solved separately for the CSL [15] $\left(\eta_{D 0}=0\right)$ and the 2SC/CFL [10] $\left(\eta_{D 1}=0\right)$ phases, see [16] for a recent review. Each subphase is locally color neutral, and the mixture is charge neutral and $\beta$-equilibrated [17]. The discussion of surface tension and charge screening effects goes beyond the present exploratory investigation. It shall be performed along the lines of Refs. [18, 19, 20].
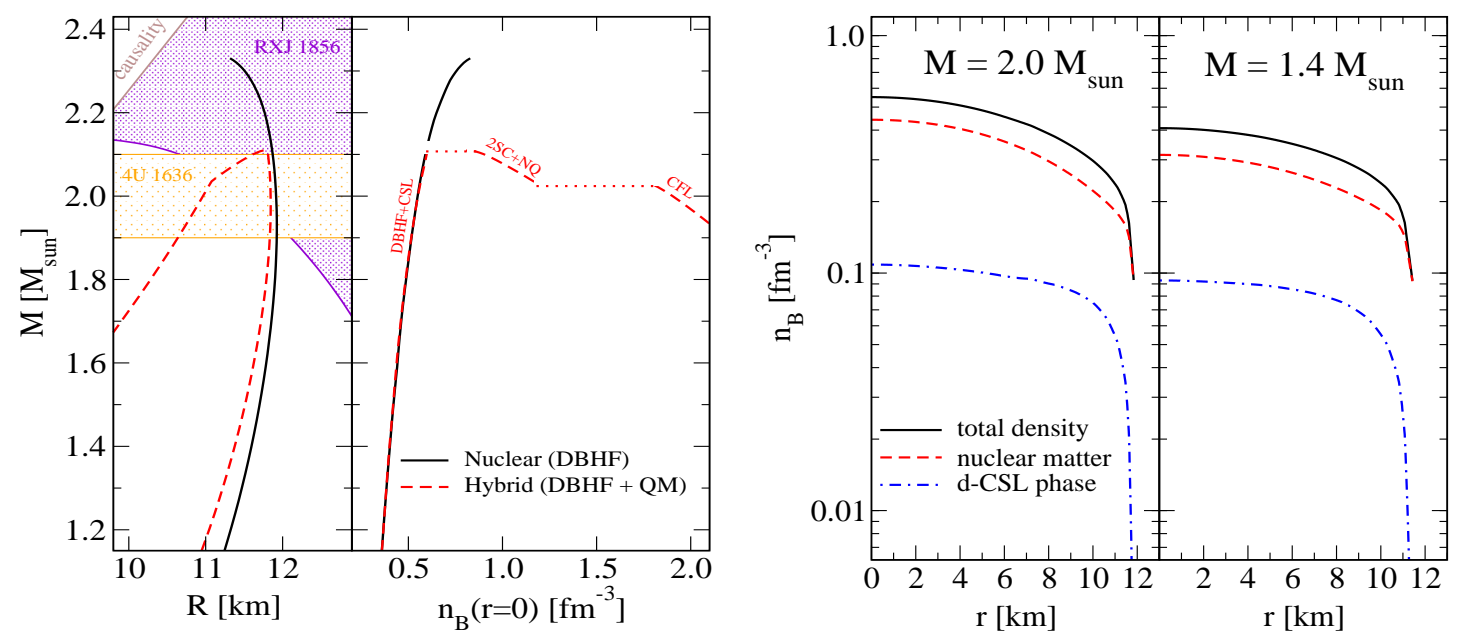

Figure 2. Left panel: Compact star sequences. The phase structure of the core changes with increasing density, as indicated in the figure. Constraints on the mass come from 4U 1636 [2] and on the mass-radius relation from RX J1856 [3]. Right panel: Density profiles of two stars with masses $1.4 \mathrm{M}_{\odot}$ and $2.0 \mathrm{M}_{\odot}$.

In Fig. 2 we show compact star sequences that correspond to the hybrid EoS described above and a $\beta$-equilibrated nuclear DBHF EoS. We also plot density profiles of two stars with masses $1.4 \mathrm{M}_{\odot}$ and $2.0 \mathrm{M}_{\odot}$. It has been suggested that matter at high densities can be probed with analyses of the cooling behavior [21, 22, 23, 24] and the stability of rapidly rotating stars against r-modes [25, 26]. Since the mixed d-quark CSL and nuclear matter phase extends up to the crust-core boundary and therefore could have significant consequences, we suggest further investigations to clarify the impact of this new phase using the probing tools mentioned above. 
Conclusions - In this contribution we have suggested a new quark-nuclear hybrid equation of state for compact star applications that fulfills modern mass-radius constraints. Due to isospin asymmetry, down-quarks may "drip out" from nucleons and form a single-flavor color superconducting (CSL) phase coexisting with nuclear matter already at the crust-core boundary in compact stars. The CSL phase has interesting cooling and transport properties that are in accordance with constraints from the thermal and rotational evolution of compact stars [27]. It remains to be investigated whether this new compact star composition could lead to unambiguous observational consequences. Eventually, it could contribute to an explanation of the puzzling superburst phenomenon [28].

Acknowledgements - We thank J. Berdermann for providing results for the CSL

phase and C. Fuchs for the DBHF EoS. D.B. is supported in part by the Polish Ministry of National Education (MENiSW). F.S. acknowledges support from the Belgian fund for scientific research (FNRS). T.K. is grateful for support from the Department of Energy, Office of Nuclear Physics, contract no. DE-AC02-06CH11357. D.B. and F.S. thank the European Science Foundation (ESF) for support from the CompStar programme.

\section{References}

[1] Klähn T et al. 2006, Phys. Rev. C 74035802.

[2] Barret D, Olive J F and Miller M C 2005, Mon. Not. Roy. Astron. Soc. 361855.

[3] Trümper J E, Burwitz V, Haberl F and Zavlin V E 2004, Nucl. Phys. Proc. Suppl. 132560.

[4] Alford M et al. 2007, Nature 445 E7.

[5] Klähn T et al. 2006, Phys. Lett. B 654170.

[6] Blaschke D B et al. 2007, Phys. Rev. C 75065804.

[7] Blaschke D, Sandin F, Klähn T and Berdermann J 2008, arXiv:0807.0414 [nucl-th].

[8] Röpke G, Blaschke D and Schulz H 1986, Phys. Rev. D 343499.

[9] Lawley S, Bentz W and Thomas A W 2006, J. Phys. G 32667.

[10] Blaschke D et al. 2005, Phys. Rev. D 72065020.

[11] Rüster S B et al. 2005, Phys. Rev. D 72034004.

[12] Abuki H and Kunihiro T 2006, Nucl. Phys. A 768118.

[13] Warringa H J, Boer D and Andersen J O 2005, Phys. Rev. D 72014015.

[14] Grigorian H 2007, Phys. Part. Nucl. Lett. 4382.

[15] Aguilera D N, Blaschke D, Buballa M and Yudichev V L 2005, Phys. Rev. D 72034008

[16] Alford M G, Schmitt A, Rajagopal K and Schäfer T 2007, arXiv:0709.4635 [hep-ph].

[17] Glendenning N K 1992, Phys. Rev. D 461274.

[18] Alford M G, Rajagopal K, Reddy S and Wilczek F 2001, Phys. Rev. D 64, 074017.

[19] Reddy S and Rupak G 2005, Phys. Rev. C 71, 025201.

[20] Voskresensky D N,Yasuhira M and Tatsumi T 2002, Phys. Lett. B 541, 93.

[21] Blaschke D and Grigorian H 2007, Prog. Part. Nucl. Phys. 59139.

[22] Popov S, Grigorian H, Turolla R and Blaschke D 2006, Astron. Astrophys. 448327.

[23] Popov S, Grigorian H and Blaschke D 2006, Phys. Rev. C 74025803.

[24] Blaschke D, Klähn T and Voskresensky D N 2000, Astrophys. J. 533406

[25] Madsen J 1999, Phys. Rev. Lett. 8510.

[26] Drago A, Pagliara G and Parenti I 2007, Astrophys. J 678 L117.

[27] Blaschke D B and Berdermann J 2007, AIP Conf. Proc. 964290.

[28] Page D and Cumming A 2005, Astrophys. J. 635 L157. 